\title{
Intracellular transport by active diffusion
}

\author{
Clifford P. Brangwynne ${ }^{1}$, Gijsje H. Koenderink ${ }^{2}$, Frederick C. MacKintosh ${ }^{3}$ and \\ David A. Weitz ${ }^{4}$
}

\author{
${ }^{1}$ Max Planck Institute for Molecular Cell Biology and Genetics, Dresden, Germany \\ ${ }^{2}$ FOM Institute AMOLF, 1098 SJ Amsterdam, Netherlands \\ ${ }^{3}$ Department of Physics and Astronomy, Vrije Universiteit, 1081 HV Amsterdam, Netherlands \\ ${ }^{4}$ Department of Physics and School of Engineering and Applied Sciences, Harvard University, USA
}

\begin{abstract}
All substances exhibit constant random motion at the microscopic scale. This is a direct consequence of thermal agitation, and leads to diffusion of molecules and small particles in a liquid. In addition to this nondirected motion, living cells also use active transport mechanisms, such as motor activity and polymerization forces that depend on linear biopolymers and are therefore fundamentally directed in nature. Nevertheless, it has become increasingly clear that such active processes can also drive significant random fluctuations that can appear surprisingly like thermal diffusion of particles, but faster. Here, we discuss recent progress in quantifying this behavior and identifying its origins and consequences. We suggest that it represents an important and biologically tunable mechanism for transport in living cells.
\end{abstract}

Thermal diffusion and random intracellular motion Diffusion is the ubiquitous random motion of small particles due to thermal agitation. Diffusive or Brownian motion was described in the early $19^{\text {th }}$ century by the botanist Robert Brown, who observed the erratic motion of particles in pollen [1]. The origin of this motion is now understood to be the thermal agitation present at the microscopic scale in any substance, which causes molecules and small particles to perform a random walk in a solution. Although diffusion is non-directional, it can be an efficient transport mechanism in cells, particularly over short distances. It is thus well-suited for transport in small prokaryotic cells, and in micro-compartments of eukaryotic cells. However, diffusive motion is also important in the cytoplasm of large eukaryotic cells, as it provides the primary basis for connectivity in signal transduction networks. Moreover, diffusive motion is of fundamental importance in developmental morphogenesis, where reaction-diffusion systems establish different embryonic domains [2].

Diffusion follows well-established physical laws: It is driven by the thermal energy of the system, and thus the diffusion coefficient that characterizes the range of motion increases with temperature, making diffusion faster. In addition, the diffusion coefficient decreases with increasing

\footnotetext{
Corresponding authors: MacKintosh, F.C. (fcm@nat.vu.nl); Weitz, D.A. (weitz@seas.harvard.edu).
}

size of the diffusing object and increasing viscosity of the surrounding medium. There are, however, a number of reports of intracellular motion that is apparently diffusive, but that does not obey these basic properties of diffusion. For example, fluorescence recovery after photobleaching (FRAP) experiments probing diffusivity of macromolecules in fibroblasts suggest that the diffusion coefficient does not exhibit the expected dependence on macromolecular size $[3,4]$. More recent studies have shown that the diffusion of inert macromolecules injected into Drosophila embryos shows a size-independent contribution to the diffusion coefficient, consistent with some additional cytoplasmic 'stirring' [5]. In some cases, motion within cells appears to be random and diffusive-like, although the magnitude of motion can be much larger than expected for thermal diffusion [6,7]. These observations suggest that there must be some other mechanism that contributes to random diffusive-like transport. The other ubiquitous transport mechanism in cells is active, directed motion, which can come from either the (de)polymerization of cytoskeletal filaments or the active motion of molecular motors along these filaments. In either case, the result is relatively straight, directed motion, such as with kinesin-driven transport of cargo along linear microtubule tracks. Although cargoes can switch between different filament tracks in a randomly oriented network [8], active, directed transport would seem to be distinct from the random motion of diffusive transport.

Here we discuss the impact of biological activity on the random, fluctuating motion of particles within cells, and suggest that such activity can contribute to random motion that is very similar in nature to thermal diffusion, resulting in an enhanced diffusivity. Thus, this behavior represents 'active diffusion.'

\section{Mechanics and diffusivity are coupled}

The random motion of particles depends not only on the driving force, be it thermal or otherwise, but also on the mechanical resistance of the surrounding medium. Thus, to fully disentangle active and thermal contributions to the motion demands a more complete understanding of cellular mechanics. This can be complicated, however, since the cell contains a heterogeneous network of cytoskeletal biopolymer filaments that gives rise to viscoelastic behavior; 
Box 1. Mechanics and fluctuating motion

The way a material responds to external forces depends on its mechanical properties. One can distinguish different kinds of materials by their time-dependent response to a shear stress. But even in the absence of external forces, the thermal motion of a particle within a material takes on a distinct time-dependence for different kinds of materials.

For a simple viscous liquid such as pure buffer or a highly dilute solution of actin filaments (Figure I a), the application of a constant force (shear stress in the left panel) leads to a deformation (shear strain) that grows with a constant rate (middle panel). A small probe particle within such a solution not only moves randomly due to thermal agitation, but the time-dependence of this motion has a very specific form: that of a random walk, in which the mean-square displacement (MSD) $\left\langle\Delta x^{2}\right\rangle$ grows linearly with time (right panel).

By contrast, in a purely elastic solid (Figure I, b), the response to an external shear stress is a (typically small) deformation that does not change in time, at least after a very short rise time (middle panel). This deformation actually results in stored elastic energy that can be recovered when the force is removed and the material returns to the undeformed state, much as a rubber band does when stretched. In such a material, thermal agitation will also give rise to motion of a probe particle. Although this motion is random, it does not correspond to a random walk, as in a viscous liquid. Rather, the motion is bounded because the restoring forces of the elastic medium do not relax or dissipate with time-a large displacement corresponds to a large energy, no matter how slowly it occurs. In this case, the MSD remains small and constant, after an initial rise (right panel).

(Figure I, c) Many materials, including networks of actin filaments under both in vitro and in vivo conditions, exhibit a viscoelastic response, being intermediate between purely viscous and elastic behavior. For a viscoelastic material subject to a constant external load, the resulting deformation of the material continues to increase, but with a decreasing rate (middle panel). Likewise, for an embedded particle in such a system, the MSD due to thermal fluctuations grows

moreover, cells typically are continually undergoing timedependent restructuring. An important starting point has thus been the study of reconstituted actin biopolymer networks and solutions in buffer, where the mechanics depends on the structural organization, including, for example, filament density and cross-linking [9-12]. At very low actin concentrations, where individual filaments do not interact, the solution behaves as a simple viscous liquid that flows continuously under a constant applied force (i.e. shear stress), since the filaments can move easily past one another (Box 1). By contrast, at very high filament concentrations, and particularly in the presence of inter-filament cross-links, filaments do not flow past one another, and the network behaves as an elastic solid. Application of a force leads to a deformation that does not increase with time. Rather, the network can store elastic energy that leads to a reversal of the deformation when the force is removed, much like a rubber band. More generally, reconstituted actin networks exhibit both viscous and elastic behavior, resulting in a slow increase in deformation upon application of a constant force. A large number of studies probing the mechanical properties of cells confirm that cells typically also behave as viscoelastic materials, and these effects cannot be ignored [13-15].

The mechanical nature of such actin filament networks is directly reflected in the thermally-induced fluctuating motion of particles within. This is best described by the mean squared displacement, $\left\langle\Delta x^{2}\right\rangle$, or the average of the square of the distance moved by the diffusing particle (Box continually, but more slowly than linearly with time. Such subdiffusive behavior is characteristic of soft, especially polymeric materials.

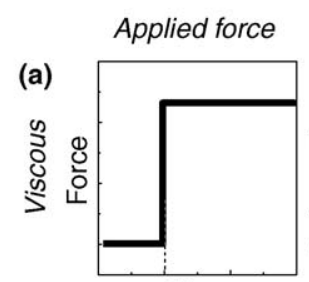

Time

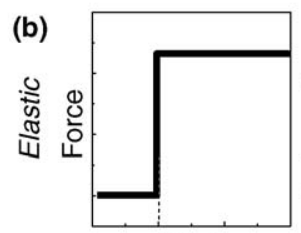

Time

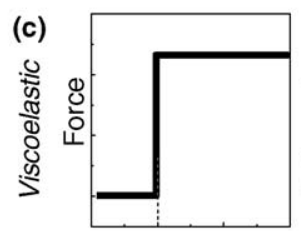

Time

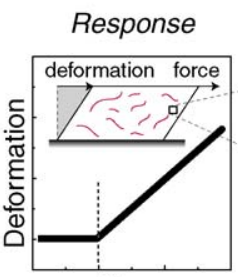

Time

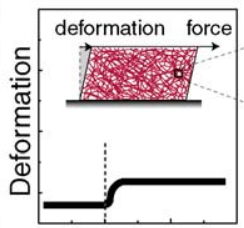

Time

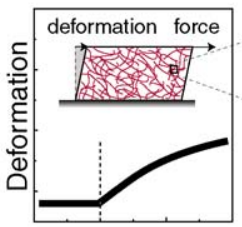

Time
Diffusive motion
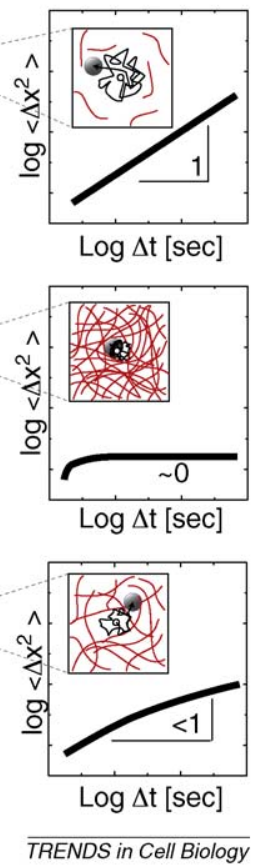

Figure I. . The nature of a material's response to applied force (e.g. shear stress) as well as fluctuations within the material (e.g. diffusive motion), depend on whether the material is viscous (a), elastic (b), or viscoelastic (c)

1). In a purely viscous solution, thermal agitation causes probe particles to exhibit a diffusive random walk, where $\left\langle\Delta x^{2}\right\rangle$ increases linearly with time. Compare this with directed motion, such as when driving along the highway: the distance traveled increases in direct proportion to the time lapsed, so the square of the distance increases as time squared. By contrast, in a dense and cross-linked reconstituted network, thermal agitation does not give rise to significant diffusive motion of an embedded probe particle; rather, the particle is stuck within the network and $\left\langle\Delta x^{2}\right\rangle$ does not increase with time. There is, however, some average motion, which reflects thermal fluctuations of the network itself, and $\left\langle\Delta x^{2}\right\rangle$ is not actually equal to zero. For viscoelastic materials between the purely viscous and purely elastic extremes, $\left\langle\Delta x^{2}\right\rangle$ increases with time, but less than linearly (Box 1). In general, fluctuating motion within viscoelastic materials can exhibit a complex time-dependence that reflects the relative importance of elasticity on different timescales.

\section{Equilibrium cell behavior}

If a cell were in true thermal equilibrium, with no motor activity or other means of creating motion, thermallyinduced fluctuations might be expected to lead to motion similar to that seen in reconstituted viscoelastic networks. The behavior expected of an inert probe particle in such a cell in thermal equilibrium is illustrated by the blue line in Figure 1. On short timescales, $\sim 1 \mathrm{~s}$, the particle senses the viscoelastic properties of the cell and its total motion is 
very small; its displacement increases slowly with time. On longer timescales the relative importance of elasticity becomes greater, the particle motion is more hindered, and $\left\langle\Delta x^{2}\right\rangle$ is then nearly independent of time. Ultimately, at longer timescales, the network behaves more liquid-like, due to structural relaxations (i.e. cross-links unbinding, filament sliding); thus, the probe particle can again move, so $\left\langle\Delta x^{2}\right\rangle$ increases. While appealing in its physical simplicity, this sort of behavior is, in fact, rarely seen in cells, primarily because of the ubiquitous activity of molecular motors and polymerization dynamics in generating additional forces and in driving the remodeling of the cytoskeleton.

\section{Insights from acto-myosin studies in vitro}

Insight into the fluctuating motion seen in cells has come through the addition of motor proteins to reconstituted networks. As also seen in vivo [16], motor activity in such reconstituted networks can give rise to unexpected behaviors that depend sensitively on the physical properties of the network, specifically the presence of cross-linking proteins. For example, several studies have shown that myosin motors can 'fluidize' un-crosslinked actin solutions, as a result of an enhancement of the ability of filaments to slide past one another [17-19]. By contrast, recent studies of cross-linked actin networks show that myosin can induce an internal tension on the network, leading to a pronounced increase in its stiffness or elasticity [20,21]. Thus, if one makes a contractile crosslinked actomyosin network in a test tube, the network not only pulls strongly against the walls (perhaps ultimately ripping away and exhibiting super-precipitation [22,23]), but in the process the network becomes much stiffer. For such a stiff elastic network, one would expect limited diffusivity of embedded tracer particles. Even in such stiffening actomyosin networks, however, the motion of tracer particles exhibits enhanced random fluctuations of a nonthermal origin. This suggests that motor activity at the level of the network need not be strictly directional [21], as expected, for example, with the randomly distributed myosin filaments at the leading edge of cells [24]. The random tracer particle motion appears to arise from uncoordinated contractile pulses exerted on the network by the myosin motors; these nonthermal pulses can lead to motion that has a time-dependence similar to that of thermal diffusion in a simple liquid, $\left\langle\Delta x^{2}\right\rangle$ increasing roughly linearly with time, but with a much higher range of motion (amplitude) [25]. This is expected in an elastic or nearly elastic material, where individual contractile fluctuations lead to step-like motion, in which the distance moved depends on both the motor force and stiffness of the network. A series of such repeated contractile fluctuations, uncorrelated with each other, can result in a random walk.

\section{Fluctuating motion and mechanics in cells}

These in vitro studies highlight the interplay between network mechanical properties and the amplitude of fluctuations within. Thus, understanding the nature of random motion in cells requires characterizing both the magnitude of the fluctuating motion, as well as the cell's inherent mechanical or viscoelastic properties. One of the

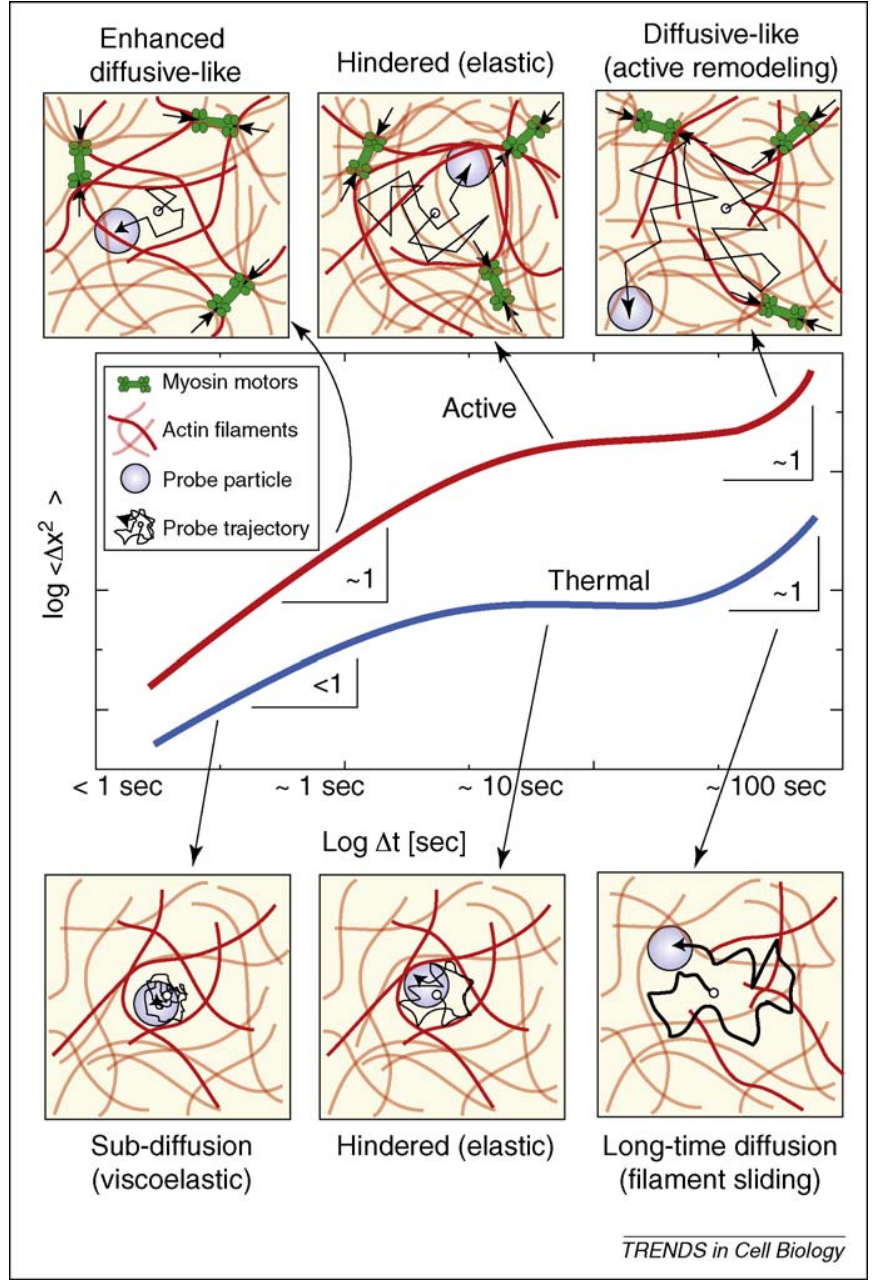

Figure 1. Active and passive fluctuating motion. If the cell were in equilibrium (non-active), the fluctuating movement, $\left\langle\Delta x^{2}\right\rangle$, of a probe particle would be expected to initially grow with time, but less than linearly, as shown by the blue curve for time scales $\sim 1 \mathrm{~s}$ (Note that $<1, \sim 1$, denotes approximate slope on a loglog plot). This is a result of viscoelasticity characteristics of cells and reconstituted actin networks (lower left panel). On longer time scales ( 10 s) the probe particle movement becomes more hindered due to increasing elastic effects (lower middle panel). On even longer time scales $(\sim 100 \mathrm{~s})$, the probe particle displacement again grows with time, because of structural relaxations (e.g. filament sliding) within the network, thereby relaxing the elastic constraints (lower right panel). By contrast, the cell appears to be actively fluctuating, with enhanced motion, as shown by the red curve. Initially, a probe particle exhibits a diffusive-like random walk (upper left panel), with $\left\langle\Delta x^{2}\right\rangle$ exhibiting a linear or slightly greater than linear increase with time. Elastic effects also slow this enhanced diffusivity on longer time-scales $(\sim 10 \mathrm{~s}$ ) (upper middle panel). On even longer time-scales, the network undergoes active remodeling and the motion again increases in time (upper right panel).

first attempts to do this was in adherent cells, where combined optical tweezer measurements of cell mechanics and passive measurements of the fluctuating behavior of probe particles suggested an active component to the random motion [26,27]. More recent combined measurements in adherent cells have shown clear evidence of a strongly active component of random intracellular motion [6,7,28], although the motion occurring on short time scales, of milliseconds or less, may still be dominated by thermal effects [29]. For example, in Dictyostelium cells, timescale separation has been used recently to extract the signatures of fluctuating microtubule motor-based activity from thermal fluctuations in the cytoplasm [30]. Similar to findings in reconstituted actin networks [21,31], these studies 
suggest that active fluctuating motion dominates on time scales longer than 10-100 ms.

The consequences of the motor activity on the fluctuations of a probe particle in a living cell are summarized schematically by the red curve in Figure 1. At early times where the total displacement remains small, $\left\langle\Delta x^{2}\right\rangle$ increases with time. The increase can even be nearly linear, similar to diffusive motion, but the amplitude of the diffusivity will be significantly larger, because the driving force is not thermal fluctuations but rather the random effects of many motor proteins. Similarly, at longer times, the motion can resemble that of an elastic network, with $\left\langle\Delta x^{2}\right\rangle$ being more nearly independent of time. The amplitude, however, is again larger than it would be for thermally-induced motion, as the fluctuations result from motor activity. Ultimately, polymerization and depolymerization of cytoskeletal filaments, as well as the dynamics of network cross-linkers, result in cell remodeling, and consequently motion of the probe, i.e., an increase of $\left\langle\Delta x^{2}\right\rangle$.

Although we focus on the random motion of embedded particles that need not be coupled directly to the network by cross-linkers or motor proteins, such direct coupling can enhance the random motion in some cases. For example, myosin-driven cargoes can exhibit diffusive-like trajectories as they hop from filament to filament in a dense random actin network [32]. Even on only one filament, cargo motion can exhibit randomness under the action of opposing motors [33]. In any case, the net motion will reflect a contribution from the specific cytoskeleton-associated process to which the cargo is coupled, in addition to nonspecific neighboring processes that can give rise to fluctuations of the cytoskeleton itself [34].

\section{Microtubules as probes of fluctuating motion}

The intrinsic connection between mechanical properties and fluctuating motion has required combined measurements of these two, as discussed above. However, we recently described a different technique to locally and non-invasively measure non-thermal forces, thereby partially overcoming this problem [31,35]. The well-characterized mechanical properties of microtubules [36,37] suggest their use as mechanical probes to measure the amplitude of cytoskeletal fluctuations. In reconstituted actomyosin networks in vitro, embedded microtubules exhibit localized bending fluctuations that were used to directly measure force pulses of order $10 \mathrm{pN}$ in magnitude in the surrounding actomyosin network [31]. The network is viscoelastic, and at low myosin concentration the microtubule fluctuations are sub-diffusive, as with thermal fluctuations. At high myosin concentrations, however, microtubule fluctuations appear to transition to a regime of surprisingly diffusive-like behavior, although this behavior is clearly myosin-driven. In living cells, microtubule fluctuations also exhibit a roughly diffusive-like behavior, but of significantly higher amplitude than expected for thermal motion, even in a simple liquid. When coupled to the in vivo dynamics of microtubule growth through an elastic surrounding meshwork, such behavior gives rise to large length-scale microtubule bends that are locked-in by the surrounding network, leading to a non-equilibrium persistence length that is about 100 times smaller than

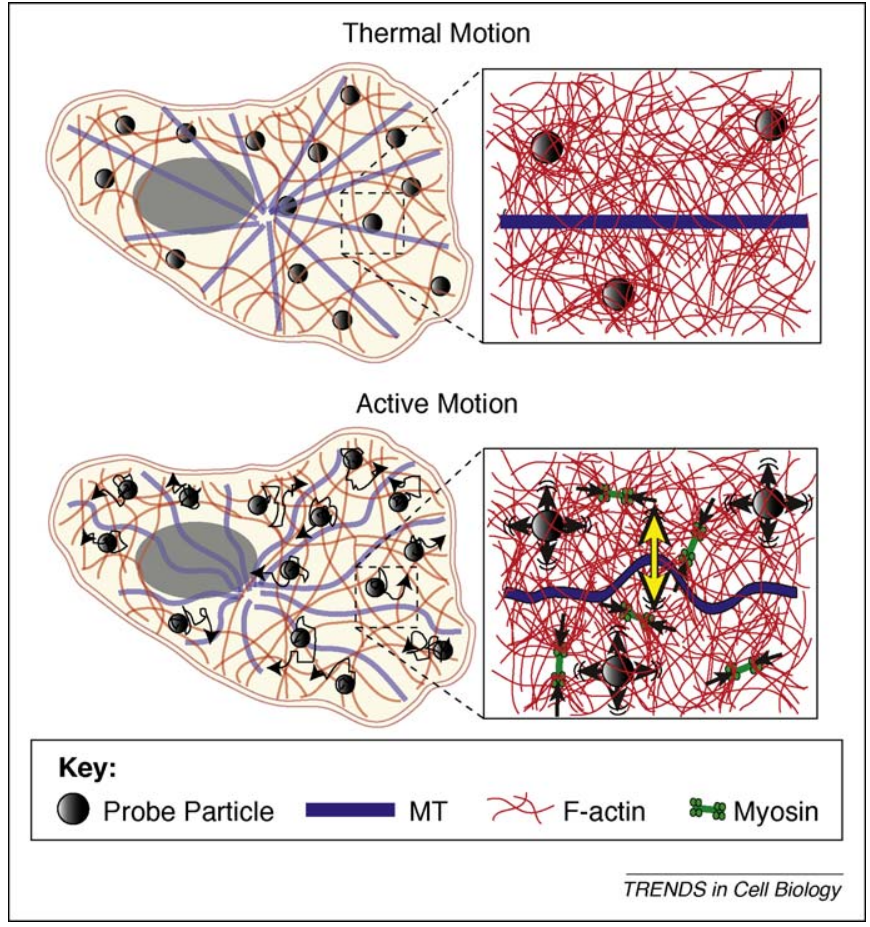

Figure 2. Active motion in cells. If the cell were in thermal equilibrium, probe or endogenous particles and organelles would undergo small-amplitude thermal motion because of the thick, viscoelastic nature of the cell. Similarly, microtubules in the cell are so stiff that thermal fluctuations of their shape would be hard to detect. By contrast, the ubiquitous activity of the motor proteins throughout the cell, and filament dynamics, lead to motion that couples the surrounding cytoplasm. This drives the motion of probe particles and all other endogenous particles, and can result in much larger amplitude fluctuating motion that can have the appearance of diffusion. Similarly, the tension in the cytoskeletal network induced by motor activity can, in turn, induce transverse forces (yellow arrow) on microtubules, leading to shape fluctuations that are sufficiently large to be detectable. If such shape fluctuations occur during the growth stage of a microtubule, they will result in large amplitude and long-length-scale bends in the shape of the microtubule, and are locked in place by the surrounding elastic medium.

that measured in vitro [35]. Similar diffusive-like, but active, microtubule fluctuations have been suggested to lead to enhanced transport of bound cargoes [34]. Microtubule behavior in living cells is influenced by interactions with other filament systems; for example, networks of actin and microtubules mutually control each other via direct mechanical linkages and by molecular signaling [38]. Even purely steric mechanical interactions between the different filament systems, however, appear sufficient for the contractile actin-myosin cytoskeleton to affect the organization and dynamics of microtubule-dependent processes in vivo [35] (Figure 2). Indeed, a steric mechanical model has shed light on the nature of microtubule load-bearing in cells [39], although the distance over which forces are transmitted depends on direct mechanical coupling [40]. In any case, the complexity of living cells calls for parallel approaches using simplified in vitro systems in combination with intracellular measurements.

\section{Concluding remarks and future perspectives}

While the active forces that drive nonthermal fluctuations in cells act primarily through the cytoskeleton, these fluctuations can have important consequences for the transport and stirring of small particles not associated with the cytoskeleton, such as organelles and perhaps even individual proteins or protein complexes. This is because of 
the fundamental hydrodynamic coupling of cytoskeletal filaments to the surrounding cytoplasm: the motion of the cytoskeleton does not occur in a stationary cytoplasmic fluid, but drags this fluid along. Thus, fluid dynamics dictates that the large, active displacements seen for microtubules must be accompanied by a very vigorous stirring and fast transport within the cytoplasm. This 'active diffusion' could be the dominant motion of individual proteins in some cases.

Much remains to be done to fully elucidate the nature and magnitude of active fluctuating motion in biological processes. However, these recent studies provide compelling evidence that active fluctuations play an important role in many processes. Moreover, although many other motors and polymerization processes can give rise to this activity, in vitro studies underscore the importance of actomyosin contractility in generating active intracellular fluctuations. By regulating the level of such motor activity, these fluctuations can be tuned to modulate the cell's own stiffness, control the rate of molecular transport by active diffusion, and even affect the dynamics of biological patterning. Motor-driven random motion has the advantage over linear transport that it is not restricted to the cytoskeletal tracks, although a random dense network of filament tracks could alternatively provide a scaffold for active diffusive-like motion. In either case, active diffusion can be much faster than thermally driven random motion. Since it can be modulated in different cells or even spatially within the same cell, active diffusion could represent a powerful mechanism for controlling gradients in signaling molecules known to be established by reaction-diffusion mechanisms. We speculate that recent puzzles surrounding the formation of morphogen gradients in development could represent examples of the influence of active diffusive motion on pattern formation in early development $[5,41]$. This suggests that the interpretation of measurements of fluctuating motion in cells must be reassessed, and the consequences of motor activity on random fluctuations must be considered to interpret properly the results of genetic and molecular perturbations of the cell.

\section{References}

1 Brown, R. (1828) On the particles contained in the pollen of plants; and on the general existence of active molecules in organic and inorganic bodies. Edinburgh New Phil. J. 358-371

2 Turing, A.M. (1952) The chemical basis of morphogenesis. Phil. Trans. Royal Soc. London Ser. B, Biol. Sci. 237, 37-72

3 Jacobson, K. and Wojcieszyn, J. (1984) The translational mobility of substances within the cytoplasmic matrix. Proc. Natl. Acad. Sci. U. S. A. 81, 6747-6751

4 Luby-Phelps, K. et al. (1987) Hindered diffusion of inert tracer particles in the cytoplasm of mouse 3T3 cells. Proc. Natl. Acad. Sci. U. S. A. 84, $4910-4913$

5 Gregor, T. et al. (2005) Diffusion and scaling during early embryonic pattern formation. Proc. Natl. Acad. Sci. U. S. A. 102, 18403-18407

6 Bursac, P. et al. (2005) Cytoskeletal remodelling and slow dynamics in the living cell. Nat. Mater. 4, 557-561

7 Lau, A. et al. (2003) Microrheology, stress fluctuations, and active behavior of living cells. Phys. Rev. Lett. 91, 198101-198104

8 Kural, C. et al. (2007) Tracking melanosomes inside a cell to study molecular motors and their interaction. Proc. Natl. Acad. Sci. U. S. A. 104, 5378-5382

9 MacKintosh, F.C. et al. (1995) Elasticity of semiflexible biopolymer networks. Phys. Rev. Lett. 75, 4425-4428

10 Janmey, P.A. et al. (1990) Resemblance of actin-binding protein/actin gels to covalently crosslinked networks. Nature $345,89-92$
11 Wachsstock, D.H. et al. (1994) Cross-linker dynamics determine the mechanical properties of actin gels. Biophys. J. 66, 801-809

12 Hinner, B. et al. (1998) Entanglement, Elasticity, and Viscous Relaxation of Actin Solutions. Phys. Rev. Lett. 81, 2614-2617

13 Fabry, B. et al. (2001) Scaling the microrheology of living cells. Phys. Rev. Lett. 87, 148102-148105

14 Bausch, A.R. et al. (1999) Measurement of local viscoelasticity and forces in living cells by magnetic tweezers. Biophys. J. 76, 573-579

15 Fernandez, P. et al. (2006) A master relation defines the nonlinear viscoelasticity of single fibroblasts. Biophys. J. 90, 3796-3805

16 Girard, K.D. et al. (2006) Dictyostelium myosin II mechanochemistry promotes active behavior of the cortex on long time scales. Proc. Natl. Acad. Sci. U. S. A. 103, 2103-2108

17 Humphrey, D. et al. (2002) Active fluidization of polymer networks through molecular motors. Nature 416, 413-416

18 Le Goff, L. et al. (2002) Motor-driven dynamics in actin-myosin networks. Phys. Rev. Lett. 88, 018101

19 Liverpool, T.B. et al. (2001) Viscoelasticity of solutions of motile polymers. Phys. Rev. Lett. 86, 4171-4174

20 Koenderink, G.H., Dogic, Z., Nakamura, F., Bendix, P.M., Mackintosh, F.C., Hartwig, J.H., Stossel, T.P. and Weitz, D.A. (2009) An active biopolymer network controlled by molecular motors. Proc. Natl. Acad. Sci. U. S. A. 2009. [Epub ahead of print, 10 August 2009].

21 Mizuno, D. et al. (2007) Nonequilibrium mechanics of active cytoskeletal networks. Science 315, 370-373

22 Hayashi, T. and Maruyama, K. (1975) Myosin aggregates as a requirement for contraction and a proposal to the mechanism of contraction of actomyosin systems. J. Biochem. 78, 1031-1038

23 Bendix, P.M. et al. (2008) A quantitative analysis of contractility in active cytoskeletal protein networks. Biophys. J. 94, 3126-3136

24 Verkhovsky, A.B. et al. (1995) Myosin II filament assemblies in the active lamella of fibroblasts: their morphogenesis and role in the formation of actin filament bundles. J. Cell Biol. 131, 989-1002

25 MacKintosh, F.C. and Levine, A.J. (2008) Nonequilibrium mechanics and dynamics of motor-activated gels. Phys. Rev. Lett. 100, 018104

26 Caspi, A. et al. (2000) Enhanced diffusion in active intracellular transport. Phys. Rev. Lett. 85, 5655-5658

27 Caspi, A. et al. (2002) Diffusion and directed motion in cellular transport. Phys. Rev. E Stat. Nonlin. Soft. Matter. Phys. 66, 011916

28 Wilhelm, C. (2008) Out-of-equilibrium microrheology inside living cells. Phys. Rev. Lett. 101, 028101

29 Van Citters, K.M. et al. (2006) The role of F-actin and myosin in epithelial cell rheology. Biophys. J. 91, 3946-3956

30 Arcizet, D. et al. (2008) Temporal analysis of active and passive transport in living cells. Phys. Rev. Lett. 101, 248103

31 Brangwynne, C.P. et al. (2008) Nonequilibrium microtubule fluctuations in a model cytoskeleton. Phys. Rev. Lett. 100, 118104

32 Snider, J. et al. (2004) Intracellular actin-based transport: how far you go depends on how often you switch. Proc. Natl. Acad.Sci. U. S. A. 101, 13204-13209

33 Welte, M.A. (2004) Bidirectional transport along microtubules. Curr. Biol. 14, R525-R537

34 Kulic, I.M. et al. (2008) The role of microtubule movement in bidirectional organelle transport. Proc. Natl. Acad. Sci. U. S. A. 105, 10011-10016

35 Brangwynne, C.P. et al. (2007) Force fluctuations and polymerization dynamics of intracellular microtubules. Proc. Natl. Acad. Sci. U. S. A. 104, 16128-16133

36 Gittes, F. et al. (1993) Flexural rigidity of microtubules and actin filaments measured from thermal fluctuations in shape. J. Cell. Biol. 120, 923-934

37 Brangwynne, C.P. et al. (2007) Bending dynamics of fluctuating biopolymers probed by automated high-resolution filament tracking. Biophys. J. 93, 346-359

38 Rodriguez, O.C. et al. (2003) Conserved microtubule-actin interactions in cell movement and morphogenesis. Nat. Cell Biol. 5, 599-609

39 Brangwynne, C.P. et al. (2006) Microtubules can bear enhanced compressive loads in living cells because of lateral reinforcement. $J$. Cell Biol. 173, 733-741

40 Das, M. et al. (2008) Buckling and force propagation along intracellular microtubules. Europhys. Lett. 84, 18003

41 Tenlen, J.R. et al. (2008) MEX-5 asymmetry in one-cell C. elegans embryos requires PAR-4- and PAR-1-dependent phosphorylation. Development 135, 3665-3675 